\title{
Evaluating Effectiveness of Modeling Motion System Feedback in the Enhanced Hess Structural Model of the Human Operator
}

\author{
Kirill Zaychik $^{1}$, Frank Cardullo ${ }^{2}$, Gary George ${ }^{3}$ \\ Man-Machine Systems Laboratory, Department of Mechanical Engineering, SUNY Binghamton \\ Binghamton, NY 13902-6000 \\ and \\ Lon C. Kelly ${ }^{4}$, \\ Unisys Corp, Hampton VA, 23666
}

\begin{abstract}
In order to use the Hess Structural Model to predict the need for certain cueing systems, George and Cardullo significantly expanded it by adding motion feedback to the model and incorporating models of the motion system dynamics, motion cueing algorithm and a vestibular system. This paper proposes a methodology to evaluate effectiveness of these innovations by performing a comparison analysis of the model performance with and without the expanded motion feedback.

The proposed methodology is composed of two stages. The first stage involves fine-tuning parameters of the original Hess structural model in order to match the actual control behavior recorded during the experiments at NASA Visual Motion Simulator (VMS) facility. The parameter tuning procedure utilizes a new automated parameter identification technique, which was developed at the Man-Machine Systems Lab at SUNY Binghamton. In the second stage of the proposed methodology, an expanded motion feedback is added to the structural model. The resulting performance of the model is then compared to that of the original one. As proposed by Hess, metrics to evaluate the performance of the models include comparison against the crossover models standards imposed on the crossover frequency and phase margin of the overall man-machine system. Preliminary results indicate the advantage of having the model of the motion system and motion cueing incorporated into the model of the human operator. It is also demonstrated that the crossover frequency and the phase margin of the expanded model are well within the limits imposed by the crossover model.
\end{abstract}

\section{Introduction}

$\mathrm{R}$ ECENT research by Cardullo ${ }^{1}$ et al (2006) significantly expanded the original Hess structural model by adding the vestibular paths as well as the models of the motion system and cueing algorithms. Figure 1 illustrates the expanded Hess model for both longitudinal and later channels. The highlighted boxes indicate the added motion feedback paths. Depending on which degree-of-freedom is being modeled, the expanded model utilizes models of otoliths or semicircular canals (SCC), which simulate motion perception in the translational and angular channels respectively. The motion feedback paths are then complemented by the motion

\footnotetext{
${ }^{1}$ Graduate student, Department of Mechanical Engineering, SUNY Binghamton, Binghamton NY, 13902, AIAA student member.

${ }^{2}$ Professor, Department of Mechanical Engineering, SUNY Binghamton, Binghamton NY, 13902, AIAA associate fellow.

${ }^{3}$ Research Associate, Man-Machine Systems Laboratory, SUNY Binghamton, Binghamton NY, 13902.

${ }^{4}$ Software Engineer, Unisys Corp, Hampton VA, 23666.
} 
platform dynamics and motion cueing algorithm. Platform dynamics is modeled by a second order transfer function (Eq.1):
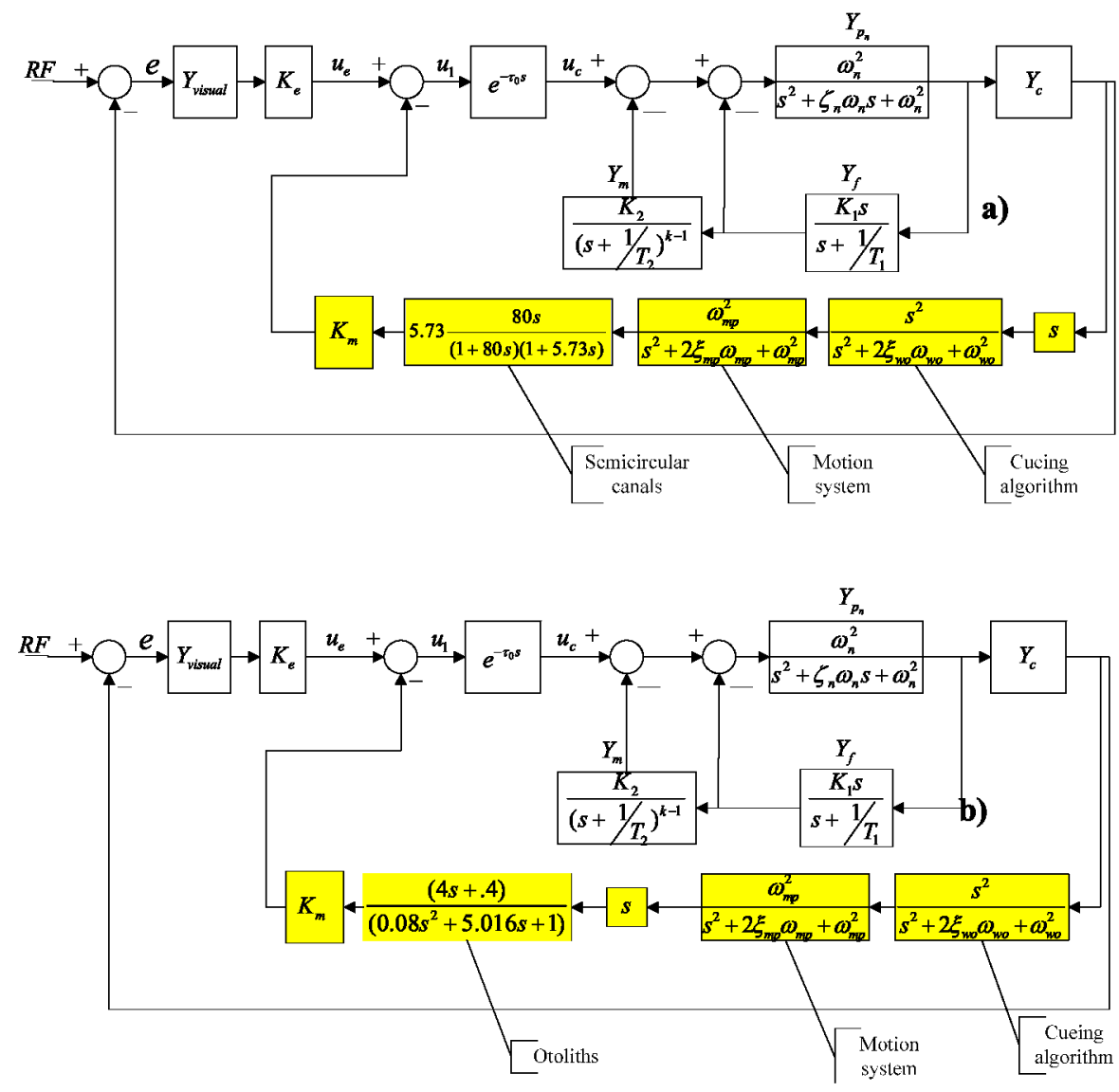

Figure 1. Expanded Hess model (Cardullo et al, 2006) with motion feedback for a) rotational channel, b) translational channel.

$$
\frac{\omega_{m p}^{2}}{s^{2}+2 \xi_{m p} \omega_{m p} s+\omega_{m p}^{2}}
$$

Where the natural frequency is $\omega_{m p}=31.4(\mathrm{rad} / \mathrm{sec})$, and the damping ratio is $\zeta_{m p}=0.7$. The washout algorithm is represented by the classical high-pass filter (2):

$$
\frac{s^{2}}{s^{2}+2 \xi_{w o} \omega_{w o} s+\omega_{w o}^{2}},
$$

With a natural frequency $\omega_{w o}=1.02(\mathrm{rad} / \mathrm{sec})$, and damping ratio $\zeta_{w o}=1.0$

The angular channel model utilizes the model of the SCC as proposed by Telban ${ }^{2}$ et al (2005): 


$$
\frac{\hat{\omega}}{\omega}=5.73 \cdot \frac{80 s}{(1+80 s)(1+5.73 s)},
$$

Where $\omega$ is the angular velocity and $\omega$ is the perceived angular velocity both in radians per second. The mathematical representation of the otoliths, used in the translational channel, was also developed by Telban et al (2005) and is given by:

$$
\frac{F_{P}}{F}=\frac{(4 s+.4)}{\left(.08 s^{2}+5.016 s+1\right)}
$$

Where $F_{p}$ is the perceived specific force and $F$ is the actual specific force resulting from the translational motion.

Parameters of all systems included in the enhanced Hess model are summarized in Table 1. Parameters inherited from the original version of the model were tuned manually so that the frequency response of the model complied with the requirements set by the crossover model in terms of the crossover frequency and phase margin.

\begin{tabular}{|c|c|c|c|c|c|c|c|c|c|c|}
\hline \multicolumn{11}{|c|}{ Hess structural model parameters } \\
\hline $\begin{array}{c}\text { Control } \\
\text { Element } \\
\text { Dynamics }\end{array}$ & $K$ & $K_{e}$ & $k_{C / O}$ & $K_{1}$ & $K_{2}$ & $1 / T_{1}$ & $1 / T_{2}$ & $\tau_{0}$ & $\zeta_{n}$ & $\omega_{n}$ \\
\hline $1 / s$ & 1.0 & 1.0 & 20.0 & 3.0 & 2.0 & 0.01 & - & 0.1 & 0.707 & 10.0 \\
\hline $1 / s^{2}$ & 1.0 & 1.0 & 20.0 & 3.0 & 2.0 & 0.01 & 0.1 & 0.1 & 0.707 & 10.0 \\
\hline \multicolumn{11}{|c|}{ Vestibular and Haptic rate feedback parameters } \\
\hline \multicolumn{2}{|c|}{$\begin{array}{l}\text { Control Element } \\
\text { Dynamics }\end{array}$} & \multicolumn{3}{|c|}{$K_{S C C}$} & \multicolumn{3}{|c|}{$K_{\text {Otolith }}$} & \multicolumn{3}{|c|}{$K_{\text {Haptic }}$} \\
\hline \multicolumn{2}{|c|}{$1 / s$} & \multicolumn{3}{|c|}{0.1} & \multicolumn{3}{|c|}{0.1} & \multicolumn{3}{|c|}{0.1} \\
\hline \multicolumn{2}{|c|}{$1 / s^{2}$} & \multicolumn{3}{|c|}{0.1} & \multicolumn{3}{|c|}{0.1} & \multicolumn{3}{|c|}{0.1} \\
\hline
\end{tabular}

Table 1. Parameters of the expanded version of the Hess structural model

The overall performance of the model with added sensory modalities was considered to be satisfactory: the phase margin with added feedback sensory data was improved, which was supported by the experimental data Cardullo et al (2006).

One of the main goals of this investigation was to automate the process of tuning/identifying parameters of the Hess structural model with the emphasis on control behavior of a given individual. This paper addresses this issue by presenting the description of the Automated Parameter Identification algorithm (APID) as well as the description of the application of APID to aid evaluating the effectiveness of the motion/vestibular system feedback of the expanded Hess structural model. 


\section{Methodology}

The methodology used during this investigation to evaluate effectiveness of the motion/vestibular system feedback is based on evaluating the ability of the Hess structural model to simulate the behavior of an actual pilot with and without the motion/vestibular feedback. First, APID is applied to identify parameters of the original version of the Hess model, in order to get as close as possible to the actual control behavior of a given pilot. In the second step the motion system is added. The resulting change in performance is evaluated by comparing PSD data of the actual and simulated control signals. During the third step of the methodology, APID is applied to the expanded version of the Hess model, where gain $K_{m}$ is identified along with other parameters of the Hess structural model. The performance of the optimized expanded version of the model is then evaluated again by comparing the PSD data of the simulated control signal against that obtained in the previous step.

\section{Automated parameter identification algorithm}

The Automated Parameter Identification algorithm can be described as an automated optimization algorithm (Figure 2), which is searching for values of the identified parameters resulting in a maximum match between the actual and modeled operator control signals. The measure of "closeness" will be referred to as fitness of a particular combination of identified parameters of the model. The APID uses a genetic algorithm (GA) based optimization engine. This choice is dictated by several factors, which include: high rate of convergence, ability to deal with highly non-linear systems, and the ability to optimize a large number of parameters, which may or may not be related to each other.

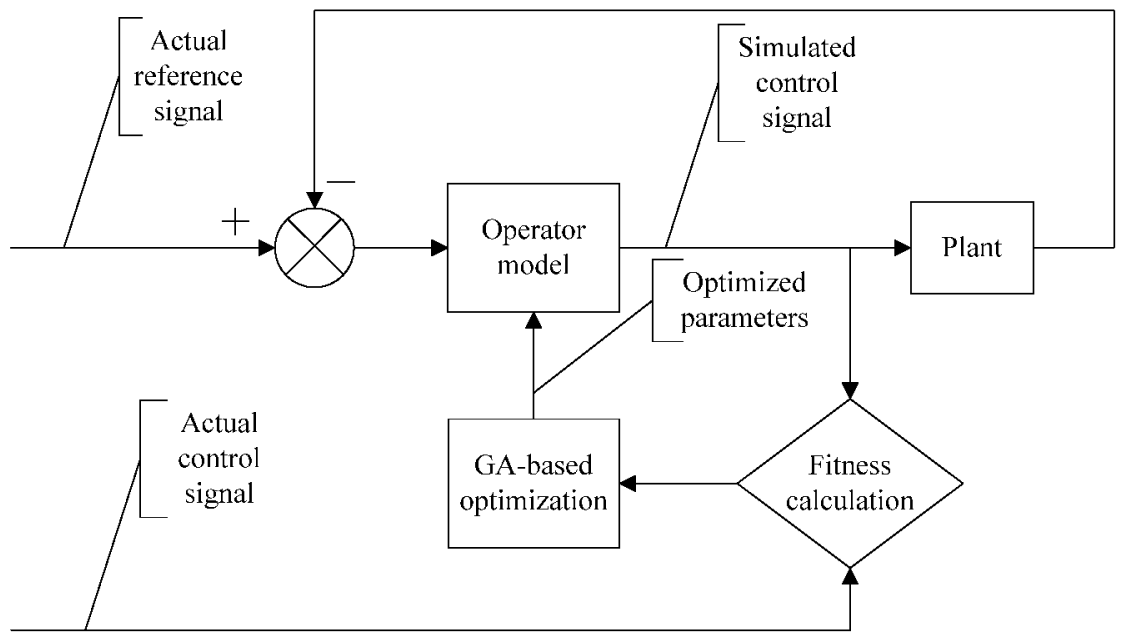

Figure 2. General architecture of the proposed APID

\section{A. Bit-Climbing Genetic Algorithm}

This section describes in detail one of the implementations of a classical genetic algorithm, namely the bitclimbing algorithm (BCGA), which was chosen to be used in APID. It was developed by Davis ${ }^{3}$ (1991) and can be described as a modified hill-climbing algorithm with certain features adapted from the classical genetic algorithm. The BCGA uses binary representation of the string of estimated parameters and/or variables similar to the classical GA. However, unlike classical genetic algorithms, it requires only a single chromosome for operation. The name "bit-climber" is inspired by the fashion in which the algorithm manipulates individual bits of the chromosome. Davis discovered that BCGA converges to a solution from 3 to 23 times faster than a traditional GA, while maintaining an acceptable accuracy. Since computational efficiency is crucial for this research it was decided to use the BCGA instead of SGA or any other traditional GA. This was verified by implementing both BCGA and SGA in APID during preliminary research. 


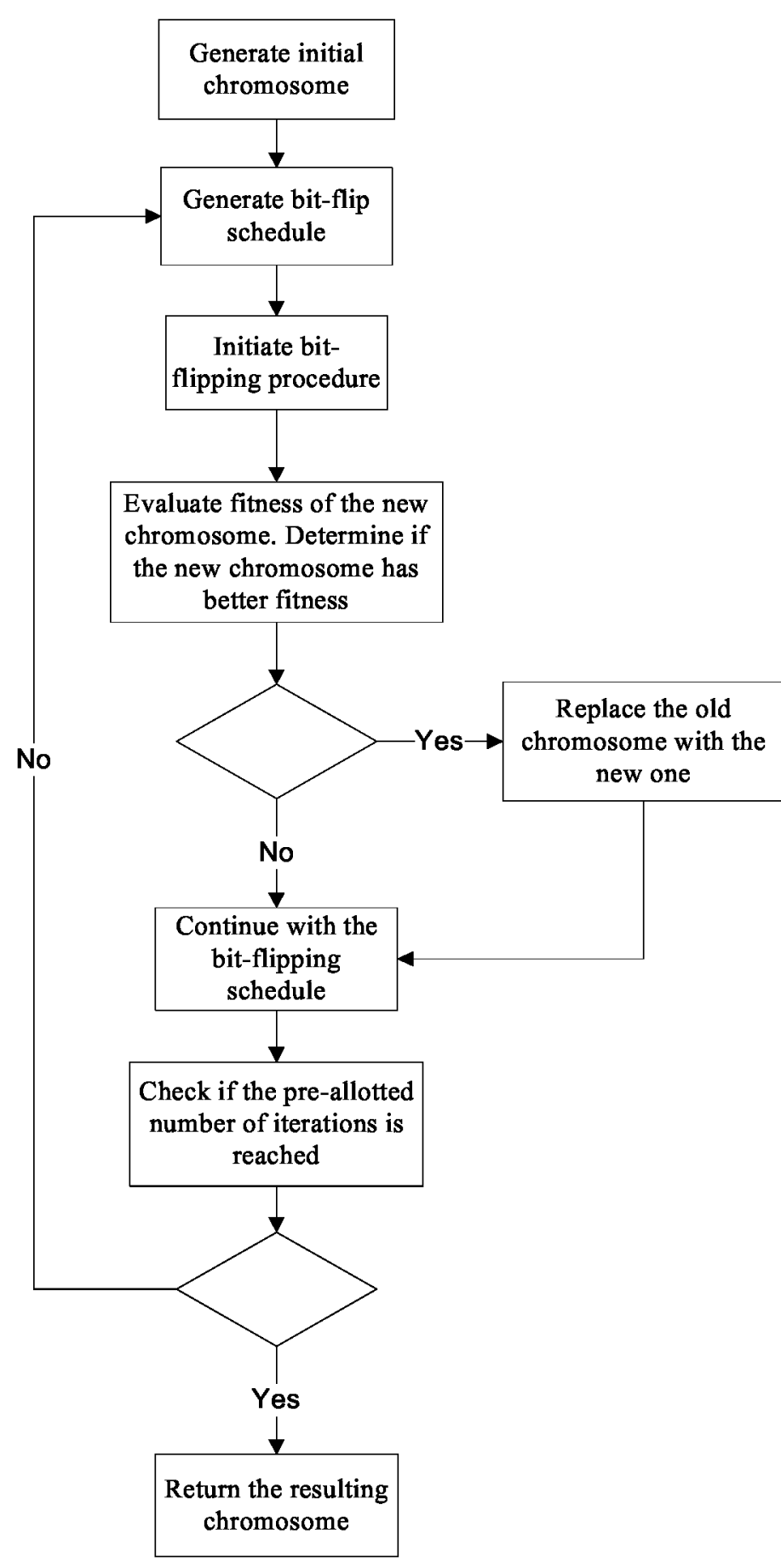

The bit-climbing algorithm begins with constructing the initial chromosome. Unlike the classical GA, the BCGA uses only a single chromosome, rather than a generation of chromosomes. On the other hand, the BCA chromosome is composed of identified parameters, put in a binary string, similarly to the classical GA. The initial chromosome is generated arbitrarily, i.e. there is no a-prior or "best guess" knowledge of the values of the identified parameters. Each identified parameters occupies 10 to 11 bits of the chromosome, depending on the desired accuracy. If, for example, the chromosome contains seven identified parameters and all parameters occupy 10 bits - it will consist of total of 70 bits. Once the initial chromosome is generated the iterative process begins by "flipping" (changing values from zero to one and vise-versa) bits of the chromosome in some arbitrary order. At each bit flip the fitness of the chromosome is calculated. Every time new chromosome produces a "better" fitness, the old chromosome is replaced with the new one and the bit flipping continues. When all bits are tested, proceed with the new iteration of flipping bits. The process continues a pre-allotted number of times or until no improvement in fitness is observed. Finally the "best" chromosome is saved. Figure 3 contains the flowchart of the bit-climbing algorithm.

Every BCGA iteration of optimization is followed by the post-processing binary to decimal conversion, which may involve division by a factor of a 100 . Hence, if a given parameter occupies 10 bits in the chromosome, it can take on values between 0 and 10.23 with the precision of up to a second digit after decimal point. In order to allow parameters to take on negative values, post-processing can include subtraction of a constant. Figure 4 illustrates the post processing associated with identification of parameters of the Hess structural model.

Figure 3. Bit-climbing genetic algorithm flowchart 

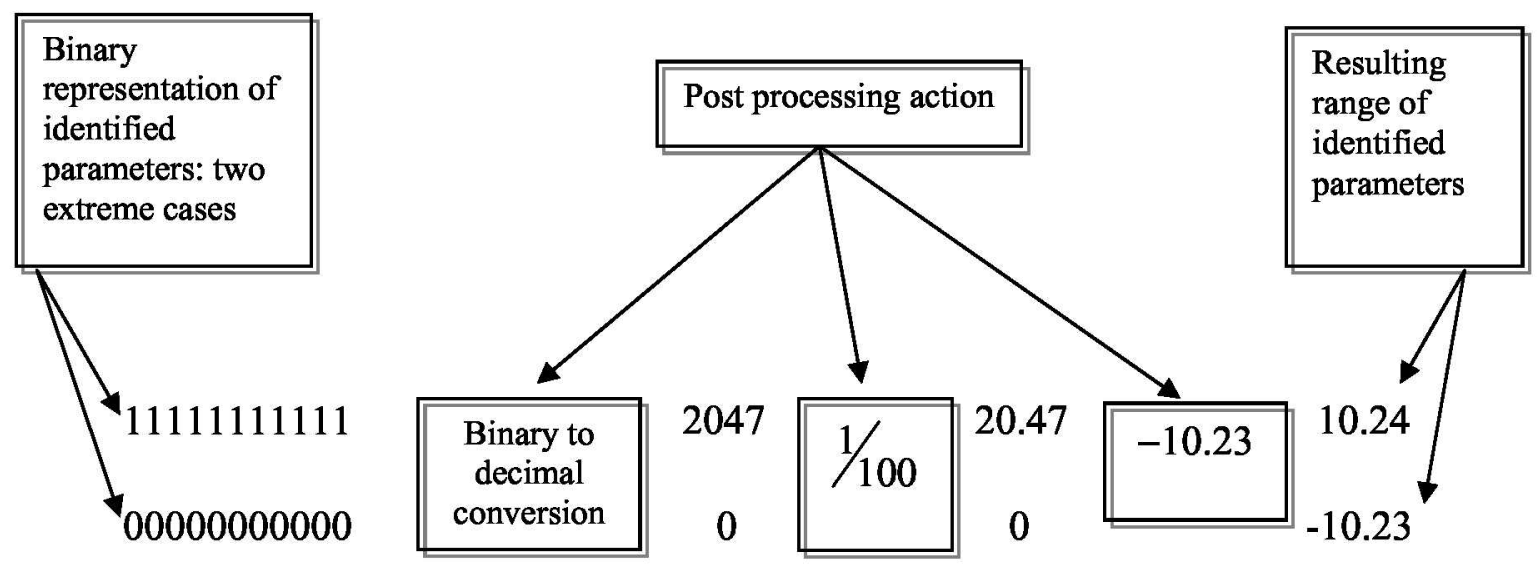

Figure 4. Pre- and post- processing associated with identification process within BCGA

The BCGA optimization routine (the outer loop of the BCGA) is repeated for 10 iterations, thus ensuring the convergence of the algorithm to a solution. It was observed, however, that the BCGA algorithm usually converges within first three iterations.

\section{B. Metrics}

This section discusses the theory and application underlining the metrics used to evaluate fitness of the chromosome generated by the BCGA. In genetic programming, fitness score can be defined as an objective measure of how close the solution is to its maximum or minimum. The term solution used here means the optimum combination of identified parameters of a given model. Therefore, fitness of the chromosome is evaluated by comparing actual and simulated control signals in the power spectrum domain. The following paragraph discusses details of computing power spectral density of the available control signals.

Power Spectral Density (PSD) of a control signal was chosen as an evaluation space for the control behavior since it allows quantifying the operator control activity in terms of the frequencies at which an individual operates as well as how much energy is spent at each frequency.

The theory of signal processing, stipulates, that it is possible to estimate the PSD of a finite length signal data set with the help of the Fast Fourier Transform (FFT). The aim of spectral estimation is to express the distribution (over the frequency range) of the power in a signal. The spectral density of a fixed random process $x_{n}$ is related to the correlation sequence by the discrete Fourier transform and is given by:

$$
S_{x}(\omega)=\sum_{x=-\infty}^{\infty} R_{x}(x) e^{-j \omega x},
$$

Where $R_{x}$ is the autocorrelation sequence and $\omega$ is the specified frequency point. Eq. 5 can be rewritten as a function of the actual frequency $f$ as follows:

$$
S_{x}(f)=\sum_{x=-\infty}^{\infty} R_{x}(x) e^{\frac{-2 \pi j f x}{f_{s}}},
$$

Where $f_{s}$ is the sampling frequency.

The PSD is then defined as: 


$$
P_{x}(f)=\frac{S_{x}(f)}{f_{s}},
$$

In order to reduce the error in spectral estimates, the method of averaging of the estimates over multiple segments of data is used. Such a method is also known as "windowing." The original time history of the control signal is divided into a series of overlapping segments. Each segment is then weighted by the type of window used. In this analysis a Hamming window was used. This choice was dictated by the fact that it is superior to a strict rectangular window due to lesser side-lobe leakage. The length of each windowed segment was chosen to contain 1024 data points. Overlapping regions contain only 32 data points. The Fourier transforms are then calculated for every weighted segment of data, which is further averaged over the resulting ensemble of PSD estimates. The resulting "smooth" spectrum estimate is then defined as:

$$
\hat{P}_{x}(f)=\left(\frac{1}{U n_{x}}\right) \sum_{k=1}^{n_{x}} \tilde{P}_{x}(f),
$$

Where $\tilde{P}_{x}(f)$ is the power spectrum computed for each data segment and $U$ is the correction factor associated with the energy loss and is equal to $0.612 ; n_{x}$ is the number of overlapping segments and is given by:

$$
n_{x}=1+\left[\frac{\left(T_{r e c}^{\prime} / T_{w i n}\right)-1}{1-x_{\text {frac }}}\right] \text {, }
$$

Where $T_{\text {win }}$ - time duration of the spectral window; $T_{r e c}^{\prime}$ - length of the extended time-history that had been filled with the trim values; $x_{\text {frac }}$ - overlap fraction $[0 ; 1]$ (the lower value corresponds to "no overlap" and the upper value corresponding to $100 \%$ overlap)

The fitness of a chromosome is computed by calculating the root mean square error (RMSE) between the two power spectrum estimates (Eq.10):

$$
E(f)=\sqrt{\frac{\sum\left(\hat{P}_{a}(f)-\hat{P}_{s}(f)\right)^{2}}{n}},
$$

In an attempt to quantify the fitness score space a benchmark score is introduced. The benchmark score is produced by executing the precision model, which is an extension of the McRuer crossover model.

\section{Control task}

This section discusses an attempt to evaluate the effectiveness of the motion system feedback using actual data

Table 2. Landing approach trim conditions. NASA LaRC VMS.

\begin{tabular}{|c|c|}
\hline Altitude & $1300 \mathrm{ft}$ BARO, 697 ft AGL \\
\hline Airspeed & $135 \mathrm{kts}$ \\
\hline Heading angle & $180 \mathrm{deg}$ \\
\hline Distance to runway & 2 nautical miles \\
\hline Flaps & Full, Gear down \\
\hline EPR & 1.19 \\
\hline Glide slope & ON \\
\hline Localizer & ON \\
\hline
\end{tabular}
obtained at NASA Langley Research Center (LaRC) Visual Motion Simulator (VMS). Actual pilot control behavior was obtained during experiments which took place at NASA Langley VMS facility and simulated the landing approach of a Boeing 757 aircraft under different control system delay conditions. One can find a detailed description of these experiments in the report by $\mathrm{Guo}^{4}$ et al (2007). In this study only the basic landing approach is 
considered, with no additional delay in the system. Table 2 contains trim conditions of the simulated aircraft.

The control task that's being investigated in this paper can be described as compensatory tracking of the roll rate of the aircraft in the presence of the lateral wind disturbance. The pilot's task was to null the roll response of the aircraft due to the lateral wind. During experiments, the wind was simulated changing its direction clockwise as shown in figure 5.a. As the wind moves to the side of the aircraft it induces a sideslip angle of the aircraft. The latter causes the aircraft to roll due to dihedral effect. The side wind is also exerting some lateral force on the vertical tail of the aircraft thus contributing to the overall rolling motion of the aircraft (Figure 5.b). The lateral component of the wind can be recorded and used as a disturbance signal, which acts upon the aircraft. The human operator in this case perceives the rolling rate of the aircraft and attempts to null the effect of such disturbance by controlling the aircraft and counteracting the wind induced rolling motion. Figure 6 contains the block-diagram of the resulting manmachine system.

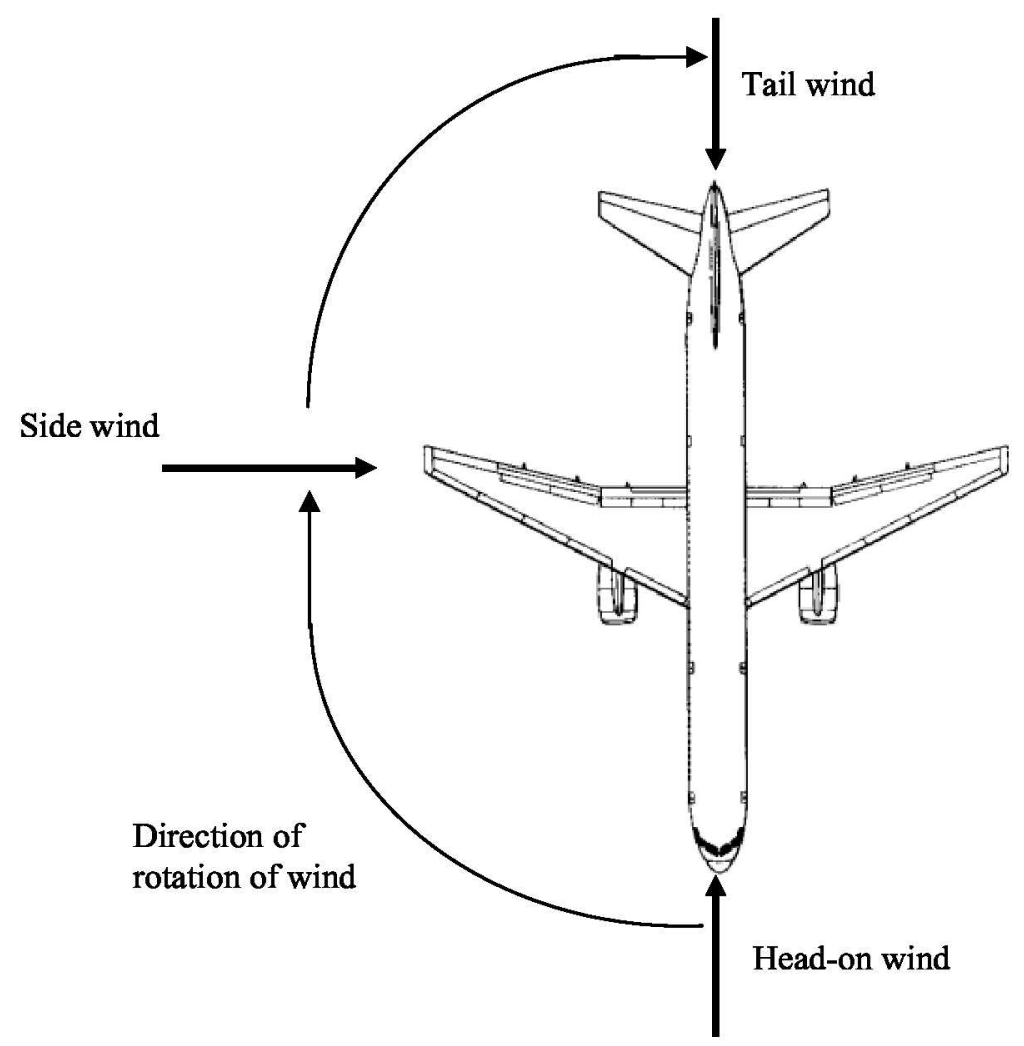

a)

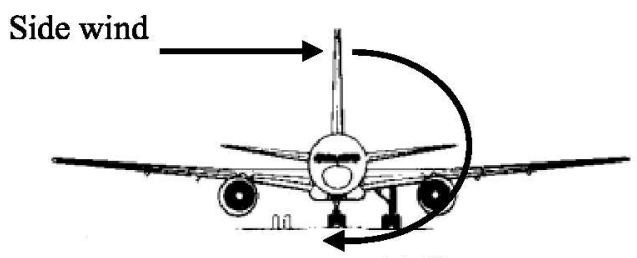

b)

Roll

Figure 5.

a) Graphical representation of the wind change pattern during the simulated landing approach.

b) Side wind acting on the vertical tail and causing the aircraft to roll 


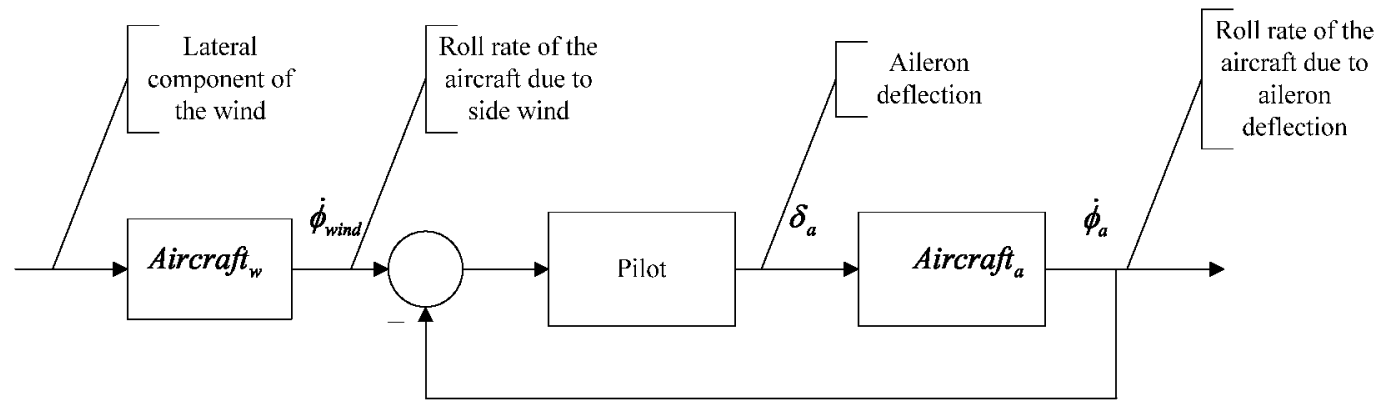

Figure 6. Block diagram of a pilot-aircraft closed loop control system used to model the stabilization task in the roll channel

In order to simulate the actual control behavior of a pilot during the lateral directional control of the aircraft it was decided to utilize a more sophisticated model of the aircraft, rather than a simple $1 / \mathrm{s}$ or $1 / \mathrm{s}^{2}$ dynamics. Roskam $^{5}$ (1979) offers a comprehensive description of the lateral directional dynamics of a wide fuselage jet aircraft. A complete transfer function relating aileron deflection $\delta_{a}$ to the roll angle $\phi$ is given by Eq.11.

$$
\frac{\phi}{\delta_{a}}=\frac{A_{\phi} s^{2}+B_{\phi} s+C}{A s^{4}+B s^{3}+C s^{2}+D s+E},
$$

Where coefficients of the numerator and denominator are the functions of the lateral-directional dimensional stability derivatives of an aircraft and are given by the systems of equation (12) and (13) respectively:

$$
\left\{\begin{aligned}
A= & U\left(1-A_{1} B_{1}\right) \\
B= & -Y_{\beta}\left(1-A_{1} B_{1}\right)-U\left(L_{p}+N_{r}+A_{1} N_{p}+B_{1} L_{r}\right) \\
C= & U\left(L_{p} N_{r}-L_{r} N_{p}\right)+Y_{\beta}\left(L_{p}+N_{r}+A_{1} N_{p}+B_{1} L_{r}\right)- \\
& -Y_{p}\left(L_{\beta}+N_{\beta} A_{1}+N_{T_{\beta}} A_{1}\right)+U\left(L_{\beta} B_{1}+N_{\beta}+N_{T_{\beta}}\right)- \\
& -Y_{r}\left(L_{\beta} B_{1}+N_{\beta}+N_{T_{\beta}}\right) \\
D= & -Y_{\beta}\left(L_{p} N_{r}-L_{r} N_{p}\right)-Y_{p}\left(L_{\beta} N_{r}-N_{\beta} L_{r}-N_{T_{\beta}} L_{r}\right)- \\
& -g \cos (\theta)\left(L_{\beta}+N_{\beta} A_{1}+N_{T_{\beta}} A\right)+\left(U-Y_{r}\right)\left(L_{\beta} N_{p}-N_{\beta} L_{p}-N_{T_{\beta}} L_{p}\right) \\
E= & g \cos (\theta)\left(L_{\beta} N_{r}-N_{\beta} L_{r}-N_{T_{\beta}} L_{r}\right)
\end{aligned}\right.
$$




$$
\left\{\begin{aligned}
A_{\phi}= & U\left(L_{\delta_{a}}+N_{\delta_{a}} A_{1}\right) \\
B_{\phi}= & U\left(N_{\delta_{a}} L_{r}-L_{\delta_{a}} N_{r}\right)-Y_{\beta}\left(L_{\delta_{a}}+N_{\delta_{a}} A_{1}\right)+ \\
& +Y_{\delta_{a}}\left(L_{\beta}+N_{\beta} A_{1}+N_{T_{\beta}} A_{1}\right) \\
C_{\phi}= & -Y_{\beta}\left(N_{\delta_{a}} L_{r}-L_{\delta_{a}} N_{r}\right)+Y_{\delta_{a}}\left(L_{r} N_{\beta}+L_{r} N_{T_{\beta}}-N_{r} L_{\beta}\right)+ \\
& +\left(U-Y_{r}\right)\left(N_{\beta} L_{\delta_{a}}+N_{T_{\beta}} L_{r}-L_{\beta} N_{\delta_{a}}\right)
\end{aligned}\right.
$$

Where $A_{1}=\frac{I_{x x}}{I_{z z}}$ and $B_{1}=\frac{I_{x z}}{I_{z z}}$. Table 3 contains expressions for the dimensional lateral-directional stability derivatives of an aircraft.

Table 3. Lateral-directional dimensional stability derivatives (Roskam, 1979)

\begin{tabular}{|c|c|}
\hline$Y_{\beta}=\frac{q S C_{y_{\beta}}}{m}, \quad\left(f t / \sec ^{2}\right)$ & $L_{\delta_{a}}=\frac{q S C_{l_{\delta_{a}}}}{I_{x x}}, \quad\left(1 / \mathrm{sec}^{2}\right)$ \\
\hline$Y_{p}=\frac{q S b C_{y_{p}}}{2 m U}, \quad(f t / \mathrm{sec})$ & $N_{\beta}=\frac{q S b C_{n_{\beta}}}{I_{z z}}, \quad\left(1 / \sec ^{2}\right)$ \\
\hline$Y_{r}=\frac{q S b C_{y_{r}}}{2 m U}, \quad(\mathrm{ft} / \mathrm{sec})$ & $N_{p}=\frac{q S b^{2} C_{n_{p}}}{2 I_{z z} U}, \quad(1 / \mathrm{sec})$ \\
\hline$Y_{\delta_{a}}=\frac{q S C_{y_{\delta_{a}}}}{m}, \quad\left(f t / \mathrm{sec}^{2}\right)$ & $N_{r}=\frac{q S b^{2} C_{n_{r}}}{2 I_{z z} U}, \quad(1 / \mathrm{sec})$ \\
\hline$L_{\beta}=\frac{q S b C_{l_{\beta}}}{I_{x x}}, \quad\left(1 / \sec ^{2}\right)$ & $N_{T_{\beta}}=\frac{q S b C_{n_{T_{\beta}}}}{I_{z z}}, \quad\left(1 / \sec ^{2}\right)$ \\
\hline$L_{p}=\frac{q S b^{2} C_{l_{p}}}{2 I_{x x} U}, \quad(1 / \mathrm{sec})$ & $N_{\delta_{a}}=\frac{q S b C_{n_{\delta_{a}}}}{I_{z z}}, \quad\left(1 / \mathrm{sec}^{2}\right)$ \\
\hline$L_{r}=\frac{q S b^{2} C_{l_{r}}}{2 I_{x x} U}, \quad(1 / \mathrm{sec})$ & \\
\hline
\end{tabular}

Where the parameter $q$ is the dynamic pressure and is equal to $q=\frac{1}{2} \rho V_{p}^{2}$

Table 4 contains values of the dimensionless lateral-directional stability derivatives as well as geometrical parameters of an aircraft and flight conditions. 
Table 4. Lateral-directional dimensionless stability derivatives (Roskam, 1979) and parameters of the aircraft

\begin{tabular}{|c|c|c|}
\hline Parameter & Value & Description (dimensions) \\
\hline$S$ & 5500 & Wing area $\left(f t^{2}\right)$ \\
\hline$b$ & 196 & Wing span $(f t)$ \\
\hline$U$ & 227 & Velocity $(f t / \mathrm{sec})$ \\
\hline$m$ & 564000 & Mass (slug) \\
\hline$\rho$ & 0.002389 & Air density $\left({ }^{s l u g} / f^{3}\right)$ \\
\hline$I_{x x}$ & $13.7 \cdot 10^{6}$ & \multirow{4}{*}{$\begin{array}{l}\text { Moments of inertia } \\
\quad\left(\text { slug } \cdot f t^{2}\right)\end{array}$} \\
\hline$I_{y y}$ & $30.5 \cdot 10^{6}$ & \\
\hline$I_{z z}$ & $43.1 \cdot 10^{6}$ & \\
\hline$I_{x z}$ & $0.83 \cdot 10^{6}$ & \\
\hline$C_{y_{\beta}}$ & -1.08 & \multirow{13}{*}{$\begin{array}{c}\text { Lateral-directional } \\
\text { dimensionless stability } \\
\text { derivatives }\end{array}$} \\
\hline$C_{y_{p}}$ & 0 & \\
\hline$C_{y_{r}}$ & 0 & \\
\hline$C_{y_{\delta_{a}}}$ & 0 & \\
\hline$C_{l_{\beta}}$ & -0.281 & \\
\hline$C_{l_{p}}$ & -0.502 & \\
\hline$C_{l_{r}}$ & 0.195 & \\
\hline$C_{l_{\delta_{a}}}$ & 0.503 & \\
\hline$C_{n_{\beta}}$ & 0.184 & \\
\hline$C_{n_{p}}$ & -0.222 & \\
\hline$C_{n_{r}}$ & -0.36 & \\
\hline$C_{n_{T_{\beta}}}$ & 0 & \\
\hline$C_{n_{\delta_{a}}}$ & 0.0083 & \\
\hline
\end{tabular}

For this research, however, the use of a reduced order aircraft transfer function (Roskam, 1979) relating roll rate $\dot{\phi}$ to aileron deflection $\delta_{a}$ was deemed to be sufficient (Eq.14). Such transfer function can be obtained as a result of "rolling" approximation of the lateral directional dynamics of the aircraft. The transfer function is derived under the assumption that there are no other lateral motion modes, such as dutch roll or spiral mode, being excited.

$$
\frac{\dot{\phi}}{\delta_{a}}=\frac{L_{\delta_{a}}}{s\left(s-L_{p}\right)},
$$

11

American Institute of Aeronautics and Astronautics 
Substituting parameters from tables 3 and 4, equation 14 can be rewritten as:

$$
\text { Aircraft }_{a}=\frac{\dot{\phi}}{\delta_{a}}=\frac{0.0107}{s(s+0.0046)}
$$

According to the block diagram shown on figure 6, knowledge of another model of the aircraft ( $A$ ircraft $t_{w}$ ), which would relate the roll rate of the aircraft to the side-wind, is required. Obtaining such model involved using the interactive Boeing 757 model provided by NASA Langley. Available aircraft (A/C) model allows simulating the behavior of the airplane in response to various pilot inputs and/or external disturbances, such as turbulence. The interface of the model allows choosing from a variety of predetermined control inputs, such as step, pulse doublet, sine wave etc. In addition to that, it is possible to feed any prerecorded, properly sampled external input as desired by the researcher. The modeled aircraft can be trimmed to virtually any flight conditions, including landing approach, cruise at low altitude, cruise at high altitude and so on. Recorded parameters match those recorded during actual simulation runs at the VMS research facility at NASA Langley research center. Availability of such interactive model of an aircraft allowed the derivation of the sought "side wind to roll rate" model by performing a standard parameter identification analysis based on the input-output data. The derivation process can be described as a four step process:

Step 1. Trim aircraft to the initial conditions corresponding to the landing approach conditions used during experiments at NASA.

Step 2. Feed the side wind external disturbance into the A/C model. Figure 7 graphically illustrates the side wind profile used during this derivation process. It is identical to that used during experiments at NASA.

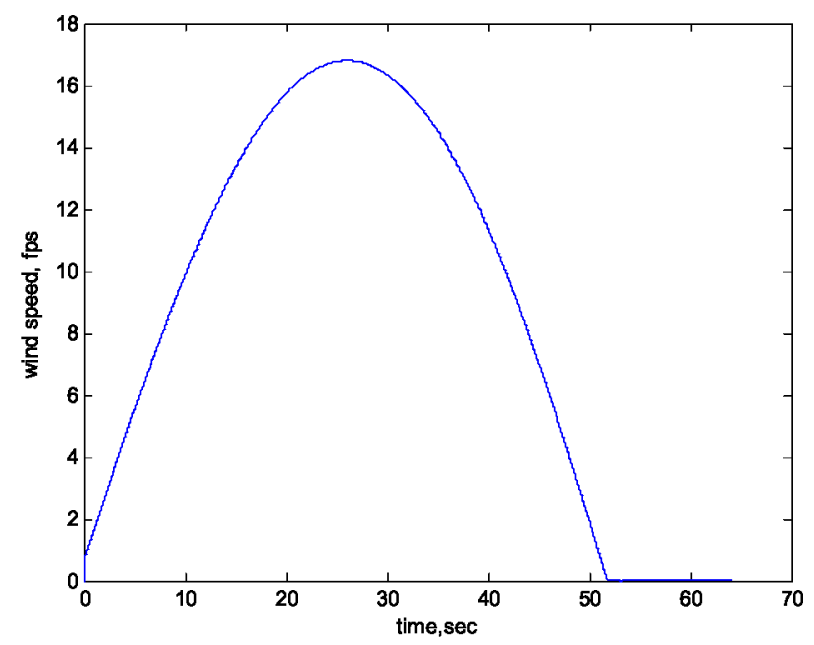

Figure 7. Lateral component of the wind acting upon the aircraft.

Step 3. Record the roll rate response of the aircraft. data.

Step 4. Using Auto Regressive with eXternal (ARX) input model estimator, fit the available input/output

With the procedure described above, it was possible to derive the following transfer function relating the lateral component of the wind acting upon the vertical tail of the aircraft to the roll rate of the aircraft (Eq. 16):

$$
\text { Aircraft } t_{w}=\frac{\dot{\phi}}{V_{\text {wind }}}=\frac{0.2554 s^{3}-14.4 s^{2}+272.6 s-25.72}{s^{4}+84.37 s^{3}+452.2 s^{2}+1510 s+628.8},
$$




\section{Results}

Figure 8 contains PSD data of four signals: the blue solid line corresponds to the actual control activity of a pilot. The dotted black line represents the power spectral density of the pilot control behavior simulated by the original Hess structural model. Parameters of the model were optimized by the APID in an attempt to closely model the pilot control behavior. It can be seen, that the model performed quite poorly throughout the entire frequency range: the major power bin was underachieved substantially.

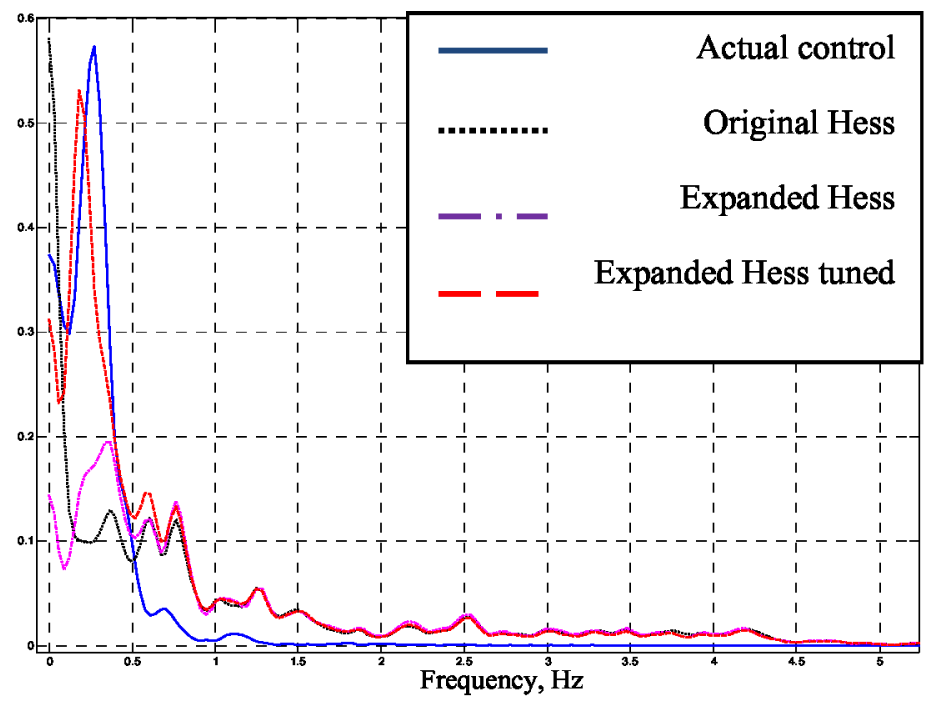

Figure 8. PSD of the actual control signal (blue solid) vs. simulated control signal by original Hess (black dotted), Expanded Hess (magenta dot-dashed) and Expanded Hess tuned by APID (red dashed).

Magenta colored dash-dotted line corresponds to performance of the Hess model with the motion feedback added into the system. Note, that the gain $K_{m}$ used in the model is equal to 150 , which is much greater than the original value proposed by Cardullo et al (2006). If the original value of 0.1 is used, then there is hardly any evidence of presence of the motion system feedback. At the initial stages of the investigation it has become clear that the gain $K_{m}$ must be increased in order to achieve some effect on the performance of the model. As one can see from the graph, the increase in gain $K_{m}$ resulted in a noticeable change of the power spectral profile of the control signal: the power level around the main power bin of the actual control signal started to elevate. In the meantime, there has been no significant alterations of the power profile in the remainder of the spectrum. This observation suggests that inclusion of gain $K_{m}$ into a string of parameters identified by APID may improve the overall performance of the model. The fourth curve on the graph (red dashed curve), corresponds to the case when parameters of the Hess structural model were identified along with the gain of the motion system feedback. It is clear from the graph, that improvement was significant. The frequency $(0.3 \mathrm{~Hz})$ of the major bin of the actual pilot control signal was estimated very closely. Moreover, the power level was matched quite accurately as well. In the mean time, there has been no change in power spectral profile past $0.8 \mathrm{~Hz}$ - it remained slightly elevated compared to the actual control signal. Table 5 contains numerical values of identified parameters of the Hess model for three cases considered. One can see only a slight improvement of the fitness score when the motion system feedback was added. The major improvement of the fitness score followed after APID had been applied to re-identify parameters of the Hess model including $K_{m}$. 
Table 5. Fitness scores produced by the original Hess structural model with and without the motion system feedback.

\begin{tabular}{|c|c|c|c|c|c|}
\hline $\begin{array}{c}\text { Model } \\
\text { configuration }\end{array}$ & \multicolumn{4}{|c|}{$\begin{array}{c}\text { Identified parameters of the } \\
\text { model }\end{array}$} & \multirow{2}{*}{ Fitness } \\
\cline { 2 - 5 } & $K_{e}$ & $K_{1}$ & $K_{2}$ & $K_{m}$ & \\
\hline Orig. & 16.2 & 8 & 2.08 & $\mathrm{X}$ & 0.0490 \\
\hline Exp. & 16.2 & 8 & 2.08 & 150 & 0.0483 \\
\hline Exp. & 7 & 3.35 & 0.95 & 976 & 0.0263 \\
\hline
\end{tabular}

Application of the above described procedure to the control data from other pilots, who participated in the experiments demonstrate an average of $50 \%$ improvement in fitness score associated with introduction of the motion/vestibular system feedback along with re-tuning of the parameters with APID.

\section{Conclusions}

This study was primarily aimed at evaluating the effectiveness of the motion/vestibular feedback developed by Cardullo et al (2006) for the Hess structural model of the human operator. The presented evaluation procedure utilizes a novel automated parameter identification technique (APID), which was designed specifically to aid in identification of parameters of a given model of the human operator. APID identifies parameters, which produce the closest approximation of actual operator control behavior. The uniqueness of the proposed APID is in using a genetic algorithm based optimization engine to achieve such a goal. The choice was dictated by the versatility and computational efficiency of genetic algorithms. There is also no obvious restriction on the number of identified parameters as well as there is no explicit requirement for them (parameters) to be related or influence each other.

The proposed evaluation procedure was based on applying APID to tune parameters of the Hess model in order to model the control behavior of a certain individual. The actual data used during evaluation was obtained at NASA LaRC VMS research facility. Experiments were conducted with the active motion system. It was demonstrated that the Hess structural model is capable of close approximation of the actual control activity when it is enhanced by the motion/vestibular system feedback. Addition of the motion system feedback resulted on average a $50 \%$ increase in model performance.

Further research in this direction may potentially contribute to answering the classical question whether one needs motion when simulating a certain control task.

\section{References}

${ }^{1}$ Cardullo, F., G. George, R. Latham, "Force Cueing Technology Integration and Feedback Metrics to Improve DMO Simulator Effectiveness", Air Force Research Laboratory, Wright-Patterson AFB, OH 45433, 2006.

${ }^{2}$ Telban, R. J., F.M. Cardullo, "Motion Cueing Algorithm Development: Human-Centered Linear and Nonlinear Approaches", Langley Research Center, NASA/CR-2005-213747, 2005.

${ }^{3}$ Davis, L., "Bit-Climbing, Representational Bias, and Test Suite Design", Proceedings of the Fourth International Conference on Genetic Algorithms, University of California, San Diego, July 13-16, 1991.

${ }^{4}$ Guo,L., F. Cardullo, L.C. Kelly, "Advanced Transport Delay Compensation Algorithms: Results of Delay Measurement and Piloted Performance Tests", NASA/CR-2007-215096, 2007.

${ }^{5}$ Roskam, J., "Airplane Flight Dynamics and Automatic Flight Controls", Part I, Roskam Aviation and Engineering Corporation, 1979. 